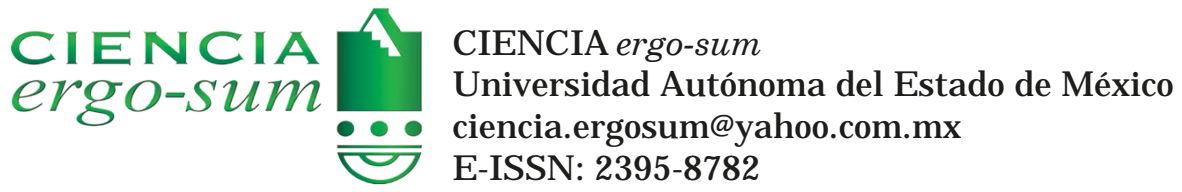

\title{
Segmentación del comprador online en México: un estudio con base en la frecuencia de compra electrónica
}

Azuela Flores, J osé I gnacio; Ochoa Hernández, Magda Lizet; Ayup González, J annett

Segmentación del comprador online en México: un estudio con base en la frecuencia de compra electrónica

CIENCIA ergo-sum, vol. 26, núm. 2, julio-octubre 2019|e47

Universidad Autónoma del Estado de México, México

Esta obra está bajo una Licencia Creative Commons Atribución-NoComercial-SinDerivar 4.0 Internacional.

Azuela Flores, J . I., Ochoa Hernández, M. L. y Ayup González, J . (2019). Segmentación del comprador online en México: un estudio con base en la frecuencia de compra electrónica. CI E NCIA ergo-sum, 26(2).

https://doi.org/10.30878/ces.v26n2a1 


\title{
Segmentación del comprador online en México: un estudio con base en la frecuencia de compra electrónica
}

Online-buyer Segmentation in Mexico: a study based on online purchase frequency

\author{
José Ignacio Azuela Flores \\ Universidad Autónoma de Tamaulipas, México \\ iazuelaf@docentes.uat.edu.mx \\ Magda Lizet Ochoa Hernández \\ Universidad Autónoma de Tamaulipas, México \\ mlochoa@docentes.uat.edu.mx \\ Jannett Ayup González \\ Universidad Autónoma de Tamaulipas, México \\ yanethay@uat.edu.mx
}

Recepción: 22 de agosto de 2017

\section{Resumen}

Se examinan los patrones de consumo en internet. Con una muestra de 1495 consumidores online, se exploran las distintas agrupaciones (tipologías) con base en su frecuencia de compra. Se empleó el análisis clúster para clasificar a los compradores mexicanos en los distintos segmentos de mercado. Los resultados muestran tres diferentes segmentos: $a$ ) frecuentes, $b$ ) ocasionales y $c$ ) esporádicos. Por medio de las tablas de contingencia y de correspondencias se analizó si las diferencias se pueden atribuir a las experiencias negativas relacionadas con la entrega del bien adquirido que experimentan los consumidores.

PALABRAS ClaVE: segmentación, análisis clúster, comprador electrónico, tipología del consumidor electrónico, frecuencia de compra electrónica.

\begin{abstract}
This article examines consumption patterns over the Internet. With a sample of 1,495 electronic consumers (National Survey on the Availability and Use of ICT in Households -ENDUTIH, 2015-), this work explores the different groups (typologies) of consumers based on their frequency of electronic purchase. Cluster Analysis was used to classify Mexican consumers in the different market segments. The results show three different segments of electronic consumers: $a$ ) frequent, $b$ ) occasional and $c$ ) sporadic. In addition, it was analyzed whether differences in consumption can be attributed to the negative experiences perceived by consumers being these related to the delivery of the acquired good through the analysis of Tables of Contingency and Correspondences.

KEYWORDS: segmentation, cluster analysis, online buyers, typology of online buyers, frequency of online purchase.
\end{abstract}

\section{INTRODUCCIÓN}

Internet como canal de venta ha generado oportunidades de mercado para las empresas, así como un cambio en los hábitos de compra de los consumidores (Ruiz Mafé y Sanz Blas, 2005).

La venta electrónica ha crecido año con año y también ha cautivado la atención tanto de académicos como directores de marketing. Por situar su magnitud, en 2016 el volumen de ventas del comercio electrónico a lo largo del mundo alcanzó la cifra de 1.86 trillones de dólares. Los pronósticos señalan que esta cifra crecerá hasta los 4.48 trillones en 2021 (Statista, 2017). En México los ingresos de las ventas en línea han ido y continúan en crecimiento. Los resultados obtenidos de la Asociación Mexicana de Internet sobre el comercio electrónico (AMIPCI, 2017) reflejan un incremento de 34\% durante el periodo de 2013 a 2014 y de 59\% entre 2014 y 2015 . De acuerdo con esta asociación, el mercado del comercio electrónico seguirá en aumento debido a que siete de cada diez usuarios de internet realizó al menos una compra en línea durante el periodo 2016. 
El crecimiento y la magnitud del mercado online exigen tanto a académicos como a directores de marketing conocer quiénes y cómo son los consumidores que realizan compras electrónicas, es decir, conocer al segmento de los compradores en línea. La segmentación de los compradores en línea contribuye a un mejor entendimiento de las características y comportamientos de estos compradores y permite a los distribuidores diseñar estrategias de marketing acordes con ello (Raju et al., 2006).

Desde el punto de vista de las empresas, considerar las características distintivas del segmento de compradores en línea es fundamental para una distribución óptima de los gastos de marketing. Una tipología del comprador en línea permitirá identificar las particularidades del segmento permitiendo a las empresas adaptar sus ofertas (Rohm y Swaminathan, 2004; Raju et al. 2006; Aljukhadar y Senecal, 2011).

La investigación previa ha utilizado diferentes variables para caracterizar al consumidor electrónico. La mayoría de estos estudios han clasificado a los consumidores electrónicos con base en indicadores sociodemográficos (Bigné et al., 2005; Chong, 2013; Bhatnager y Ghose, 2004; Donthu y Garcia, 1999; Kau et al., 2003; Vrechopoulos et al., 2001), indicadores motivacionales (Rohm y Swaminathan, 2004) e indicadores psicográficos (Barnes et al., 2007; Cristóbal-Fransi et al., 2014). En este sentido, las características sociodemográficas son las más empleadas dentro de la segmentación de los consumidores electrónicos (Rodríguez-Torrico et al., 2012), ya sea como variable base de segmentación (Bigné et al., 2005; Chong, 2013) o para conocer más acerca del perfil del comprador (Bhatnagar y Ghose, 2004; Yang y Hye-Young, 2012).

Sin embargo, y a pesar de la abundante literatura sobre segmentación del consumidor electrónico y de la importancia de la frecuencia de compra que pronostica la intención de compra electrónica (Hernández et al., 2011), muy pocos trabajos han caracterizado a los compradores con base en variables comportamentales, a excepción del trabajo de Chen et al. (2009) que clasificó a los compradores electrónicos en segmentos con base en la frecuencia de compra.

La clasificación de los compradores electrónicos con base en la frecuencia de compra a través de internet no sólo ayuda a un mejor entendimiento del comportamiento de consumo a través de este canal, sino que contribuye a la literatura existente sobre tipologías de consumidores electrónicos. La segmentación de compra online con base en la frecuencia favorece el pronóstico de la intención de compra electrónica (Hernández et al., 2011), pero también explica parte importante de las ventas electrónicas. La aportación al volumen de ventas es mayor en el caso de los compradores frecuentes respecto a los infrecuentes (Min et al., 2012; Chiou y Pan, 2009). Además, los consumidores frecuentes no sólo incrementan las ventas, sino que también manifiestan mayores niveles de satisfacción y lealtad hacia el minorista electrónico (Chiou y Pan, 2009). Por lo anterior, comprender las diferentes necesidades de compradores, tanto frecuentes como esporádicos, es importante para lograr la fidelidad del cliente (Chiou y Pan, 2009; Joia y Sanz, 2006). Por tanto, el objetivo de este artículo es precisamente el de segmentar a los compradores online con base en la frecuencia de consumo en internet.

Una vez caracterizados los compradores electrónicos, el interés se centra en analizar las diferencias de consumo electrónico entre los segmentos identificados y, en particular, el efecto de las experiencias en la compra en línea. De acuerdo con la literatura, las experiencias pueden modificar la intención de compra (Becerra y Korgaonkar, 2011; Klaus, 2013; Pappas et al., 2014). La investigación previa ha revelado que las experiencias satisfactorias (positivas) influyen positivamente en la intención de compra (Pappas et al., 2014; Zhou et al., 2007), mientras que las experiencias insatisfactorias (negativas) influyen negativamente (Anderson y Srinivasan, 2003). En este tenor, el artículo hace un análisis del efecto de las experiencias negativas sobre la frecuencia de compra electrónica, es decir, si las diferencias en el consumo pueden atribuirse a las experiencias previas negativas experimentadas por los consumidores.

Posteriormente, se presenta una revisión analítica de la literatura revisada sobre las diferentes tipologías de consumidores electrónicos, y después se describen los datos y la metodología empleada. Enseguida se exponen los resultados y se describen los segmentos identificados. El trabajo finaliza con la discusión de los resultados y conclusiones, así como las propuestas para estudios futuros. 


\section{Tipologías DEL CONSUMIDOR ELECTRÓNICO}

El desarrollo de tipologías de compradores en línea es una corriente de investigación bien establecida que ha utilizado una variedad de bases tales como motivaciones de compra, actitudes hacia la compra, lealtad a la tienda, estilos de vida, entre otros. Así pues, Vrechopoulos et al. (2001) segmentan dos grupos de consumidores electrónicos a través de medidas demográficas, características comportamentales, percepciones y preferencias, y también destacan que ambos grupos representan en su mayoría a hombres jóvenes con un nivel educativo y de ingresos alto. Respecto a sus características comportamentales, ambos grupos prefieren comprar por internet debido a la posibilidad de compra las 24 horas y el ahorro de tiempo. En cuanto a las percepciones y preferencias, ambos grupos compran hardware y software, discos, libros y revistas, vuelos y electrónica; sin embargo, uno de los grupos valora más un servicio de alta calidad y de entrega rápido, mientras que el otro valora la amplia variedad de productos.

Por su parte, el estudio de Liu et al. (2015) segmenta a partir del comportamiento de compra: a) economical purchasers (son sensibles al precio y en general les lleva tiempo tomar sus decisiones de compra), b) active-star purchasers (les gusta comunicar sobre sus experiencias de compra), c) direct purchasers (los que tienen objetivos claros respecto a lo que van a adquirir y toman tal decisión en el corto plazo), d) high-loyalty purchasers (se distinguen por tener preferencias claras, conocimiento del producto y experiencia en la compra) ye) credibility-first purchasers (aquellos que tienen preferencia por ciertos establecimientos con alta reputación).

También, desde las actitudes y el comportamiento de compra, Kau et al. (2003) desarrollaron una tipología del consumidor electrónico: a) quienes gustan de navegar en internet y poseen experiencia en la red, pero prefieren comprar fuera de línea (on-off shopper), b) quienes comparan características del producto, precios y marcas antes de tomar las decisiones de compra (comparison shopper), $c$ ) aquellos que siguen comprando en tiendas fuera de línea y no les gusta navegar por internet ni buscan comparar precios (traditional shopper), $d$ ) quienes gustan de comparar marcas y características de los productos, incluso confían en el internet para recopilar información, pero no están particularmente propensos a realizar la compra (dualshopper $), d)$ aquellos que presentan bajo interés en la búsqueda de información vía internet (e-laggard) y e) quienes buscan promociones, ofertas, tienen experiencia en la red y la compra en línea, les gustan los banners (anuncios) y dan click en ellos con frecuencia (information surfer).

A partir de la integración de dimensiones de personalidad y de factores culturales, Barnes et al. (2007) distinguen tres segmentos de clientes: a) los extremadamente cuidadosos, reservados y usualmente escépticos a nuevas experiencias, la mayoría de ellos nunca ha comprado a través de internet. $b$ ) Los que muestran menor riesgo percibido a las compras en línea, una actitud positiva, alta disposición de compra y confianza hacia las ventas por internet. c) Los que se muestran cuidadosos y reservados, con alto riesgo percibido al comprar en internet a pesar de tener una actitud positiva, y utilizan este medio principalmente para la búsqueda de información y evaluaciones del producto antes de su compra.

Si bien la mayoría de los consumidores usa internet con regularidad, es importante resaltar que los propósitos para los cuales lo usan son distintos. Al respecto, el estudio de Aljukhadar y Senecal (2011) segmenta a los consumidores en línea con base en los diversos usos que le dan al internet: a) quienes lo utilizan sobre todo para comunicarse vía correo electrónico, $b$ ) quienes lo utilizan para navegar y comprar y $c$ ) quienes explotan las características interactivas de este medio: chat, blogs, descargas, entre otras.

Por su parte, Allred et al. (2006) clasificaron a los usuarios de internet en dos grandes grupos mediante características demográficas, psicográficas y de uso de la computadora. Los primeros se caracterizan por ser compradores activos impulsados por el deseo de socializar, jóvenes, educados, con mayor conocimiento informático, les gusta minimizar riesgos, son grandes consumidores de minoristas online e invierten mucho tiempo en la computadora. Por el contrario, el segundo grupo, a pesar de participar en diversas actividades en el internet, se resiste a comprar en línea; la inseguridad y las escasas habilidades en tecnología les impiden hacer transacciones, por lo que no resulta un segmento atractivo para el comercio minorista online. 
En relación con las características de los estilos de vida, Swinyard y Smith (2003) encontraron cuatro segmentos para los consumidores en línea: a) aquellos muy capaces para la realización de compras en línea y con pocos obstáculos al realizar la actividad; representan un mercado ideal para los vendedores, especialmente en productos como ropa, libros y música. b) Otro segmento lo componen personas versátiles y prolíficas en el uso en línea. Su actividad por medio de internet es muy alta, pues leen noticias y revistas, visitan sitios relacionados con sus pasatiempos, reservan entradas, buscan oportunidades de trabajo o descargan software, entre otras actividades. c) Un segmento cuyo uso principal es jugar videojuegos, comprar boletos o hacer reservas, y además está en proceso de aprendizaje sobre cómo utilizar internet. d) Aquellas personas que usan internet para hacer negocios y muestran un serio interés en lo que puede hacer por ellos profesionalmente.

En esta sintonía, Brengman et al. (2005) desarrollan cuatro tipologías muy parecidas a las descritas por Swinyard y Smith (2003), pues una de ellas lo integran personas que gustan de comprar en internet y disfrutan cada una de sus facetas (comodidad, ofertas, etcétera); el uso que le dan es para buscar información, diversión y explorar sitios web. Otra categoría la integran quienes confían en el uso de la computadora y utilizan internet para propósitos de competencia y negocios. Otra, aquellos menos alfabetizados respecto al uso de internet, puesto que son los más temerosos y de opinión negativa sobre la logística de internet, la oferta de compras en línea y la conveniencia; por tanto, son los que hacen el menor uso. Finalmente, quienes, a pesar de su experiencia en las compras, no están convencidos de que al hacerlo en línea provea mayor conveniencia o mejores ofertas, así como una contribución real a su vida. Este grupo no sobresale en actividades sociales, informativas, de entretenimiento o de negocio en la web.

Referente a las motivaciones de compra y a los atributos de la tienda, Ganesh et al. (2010) agrupan a los consumidores que carecen de motivación hacia alguna de las dimensiones de compra y presentan baja consistencia respecto a la importancia de los atributos, a los que se caracterizan por altos valores en todas las dimensiones motivacionales y medidas de importancia de los atributos, a aquellos vanguardistas en cuanto a las dimensiones motivacionales y en cuanto a los atributos (el atractivo del sitio web y la variedad de productos), a los motivados por la conveniencia de comprar y sin interés en la variedad de los productos y, por último, quienes están orientados al precio, disfrutan de encontrar ofertas y son proactivos en su búsqueda. A su vez, Rohm y Swaminathan (2004) desarrollan cuatro grupos: a) el primero está motivado por la conveniencia. $b$ ) El segundo, por la búsqueda de variedad a través de las distintas alternativas, tipos de productos y marcas. $c$ ) El tercero, por la conveniencia y la búsqueda de variedades. d) El cuarto, por la orientación física, es decir, por la posesión inmediata de los bienes y la interacción social.

Por su parte, Hill et al. (2013) identifican segmentos de adolescentes con base en las motivaciones de uso y compra por internet. Así, distinguen a los que tienen las más altas motivaciones sociales, de descubrimiento y escapismo en línea, pero sin gran influencia en las compras familiares, a quienes reportan altos niveles de motivaciones de utilidad, pero el nivel más bajo de motivación social y menor probabilidad de influir en las decisiones de compra familiares. Los otros son aquellos que, a pesar de presentar bajas motivaciones de uso, muestran el mayor nivel de disfrute en las compras en línea; por tanto, influyen en las decisiones del hogar al efectuar compras por este medio.

Otra de las variables utilizadas para segmentar a los clientes electrónicos es el estado afectivo, específicamente las emociones y sentimientos que los usuarios experimentan al comprar en línea. Al respecto, Christodoulides et al. (2013) distinguen seis segmentos: a) aquellos quienes se sienten más felices, cómodos, seguros, expresivos e imaginativos estando en línea. b) Los que experimentan estados afectivos más intensos al no estar conectados al internet.c) Los que experimentan el mismo nivel de emociones y sentimientos positivos, tanto en línea como fuera de ella. d) Los que tienen los puntajes más altos en todos los estados afectivos; su vida en el internet les hace sentir emociones y sentimientos más intensos que cuando no están conectados. e) Los que asignan la misma intensidad afectiva tanto al mundo real como al virtual. $f$ ) Los que se sienten más estresados, ansiosos y conservadores cuando están fuera de línea y más anónimos, aventureros, expresivos e imaginativos si sí lo están. 
De acuerdo en su preocupación por la privacidad, Sheehan (2002) determinó una tipología cuyos niveles de preocupación por la privacidad variaban con base en la situación que se presentaba: a) quienes reportan una mínima preocupación en la mayoría de las situaciones relacionadas con la privacidad, $b$ ) quienes muestran un nivel moderado de preocupación en todas las situaciones relacionadas con la privacidad, $c$ ) los que tienen un nivel moderado de preocupación en la mayoría de las situaciones relacionadas con la privacidad y $d$ ) quienes se muestran muy preocupados con la privacidad en todas las situaciones.

Si bien es cierto que clasificar a los compradores electrónicos con base en la frecuencia de compra es común entre los gerentes de marketing, desde el punto de vista académico, muy pocos trabajos han contribuido a la construcción de una tipología desde esta variable. Una excepción es la de Chen et al. (2009), quienes sí usaron esta variable (frecuencia de compra), además de la cantidad de dinero gastado en cierto periodo, así como la fecha de la última compra para proponer un método que recoja los patrones de consumo en el tiempo de la compra por internet, lo cual ofrece información valiosa para la toma de decisiones. Por su parte, Hernández et al. (2011) destacan la importancia de la frecuencia, pues pronostica la intención de compra; sin embargo, en su estudio, encuentran que las variables socioeconómicas no condicionan el comportamiento de los compradores en línea experimentados.

En el caso de Prodanova y San Martín Gutiérrez (2013), ellas contribuyen a la construcción de un perfil del consumidor turístico a través de la venta online de billetes de transporte y comprueban que existe alta frecuencia de compra, puesto que lo realizan más de tres veces al año. Wu y Chou (2011) analizan el comportamiento de compra y actitudes de los consumidores usando datos de un sitio web de compra online y proponen un método de segmentación de clientes en múltiples categorías; una de éstas engloba la frecuencia de compra y el dinero gastado en la compra. En este sentido, Martin et al. (2015) identifican dos segmentos importantes en la venta minorista electrónica: a) los compradores online frecuentes y $b$ ) los infrecuentes. Los primeros han realizado entre cuatro y seis transacciones en las últimas doce semanas previas al cuestionario y los segundos han comprado sólo una vez en ese mismo periodo. Rose et al. (2012) utilizaron la frecuencia de compra en su estudio sobre las experiencias de compra online, a partir de lo cual proponen un modelo conceptual mediante variables antecedentes directas e indirectas, de componentes y de resultado; específicamente, las variables antecedentes son formativas de los componentes cognitivos y afectivos, lo que a su vez conduce a tres comportamientos de resultado: satisfacción, confianza e intención de recompra. Por su parte, Joia y Sanz (2006) analizan la rentabilidad de las transacciones de los compradores de comercio electrónico a través de la frecuencia. Construyen una tipología de compradores frecuentes y esporádicos y concluyen que una mayor frecuencia de compra no implica necesariamente una mayor rentabilidad; con esto destaca el hecho de comprender el valor real que aporta cada cliente.

Esta revisión de la literatura muestra la heterogeneidad de usuarios en los segmentos de mercado online, los cuales siguen siendo sujeto clave de estudio toda vez que pueden ser diferentes totalmente de un segmento a otro. Como se puede constatar, de acuerdo con las tipologías presentadas, ningún estudio determina un perfil de consumidor online tomando como base de segmentación la frecuencia de consumo agrupado con dimensiones sociodemográficas y conductuales. Este tópico se desarrollará en el siguiente apartado.

\section{Datos y metodología}

Este artículo, de acuerdo con la frecuencia de consumo electrónico, se centra en establecer una tipología del consumidor mexicano en internet. Para este propósito, se emplearon los datos de la Encuesta Nacional sobre Disponibilidad y uso de TIC en Hogares (ENDUTIH) aplicada por el Instituto Nacional de Estadística y Geografía (INEGI, 2015).

La encuesta ENDUITH se aplicó a nivel nacional en una muestra de 90030 viviendas seleccionadas por medio de un muestreo probabilístico (polietápico, estratificado y por conglomerados) y distribuida en las 32 entidades federativas. La unidad de análisis se estableció a partir de los residentes habituales del hogar de seis años y más de edad. 
Del total de observaciones, se obtuvo una muestra de 1495 personas que, seleccionadas en un muestreo aleatorio sistemático, declararon haber realizado compras en internet. ${ }^{[1]} \mathrm{El}$ perfil socio-demográfico predominante de la muestra corresponde al de hombres (57\%), de 32 años en promedio y con estudios superiores ( $50 \%$ manifiesta que posee estudios de licenciatura o profesional), quienes declaran haber comprado a través de internet principalmente bienes y servicios personales (52\%), reservaciones y boletos (42\%), aparatos electrónicos (28\%), programas y aplicaciones (24\%) y bienes y servicios para el hogar (20\%).

Para segmentar al consumidor se empleó el análisis clúster. Esta técnica de agrupación asigna individuos a grupos buscando la homogeneidad dentro de éstos y, al mismo tiempo, la heterogeneidad entre los grupos de acuerdo con una o más variables indicador (Iacobucci y Churchill, 2015).

Se empleó la metodología estadística de clúster no jerárquico. Concretamente, se realizó un $K$-means (K de medias), bajo el cual las observaciones son reasignadas al clúster con el centroide más cercano y con ello minimizar la varianza dentro de cada clúster (Punj y Stewart, 1983).

\section{Resultados}

Como se dijo, para crear la tipología de consumidores electrónicos, se recurrió a una técnica de agrupación de casos (individuos) en grupos que logre la máxima homogeneidad en cada uno y la mayor heterogeneidad entre ellos. Así pues, se empleó el análisis clúster no jerárquico de aglomeración $K$-means. [2]

La solución definitiva elegida para este estudio es de tres conglomerados, en la cual se atiende el tamaño de los grupos, la posibilidad de interpretación de los resultados, el grado de significación de cada factor en cada uno de los análisis (ANOVA) y la posición de los centroides finales (cuadro 1 y cuadro 2).

\section{CUADRO 1}

Análisis clúster no jerárquico

\begin{tabular}{|lccccc}
\hline Variable indicador & Estadístico & \multicolumn{3}{c}{ Centros finales } \\
\hline & $\mathrm{F}$ & Sig. & Grupo 1 & Grupo 2 & Grupo 3 \\
\cline { 2 - 6 } $\begin{array}{l}\text { Frecuencia de compra a través de } \\
\text { internet }\end{array}$ & 16222.730 & 0.000 & 1 & 3 & 2 \\
\hline
\end{tabular}

Fuente: elaboración propia.

CUADRO 2

Descripción de los clúster

\begin{tabular}{|lcccccc}
\hline & & & Tamaño & \multicolumn{2}{c}{$\begin{array}{c}\text { Distancia entre los centros de los } \\
\text { conglomerados finales }\end{array}$} \\
\cline { 4 - 7 } Grupo & Denominación & Núm. & $\%$ & Con 1 & Con 2 & Con 3 \\
\hline 1 & Comprador frecuente & 441 & 30 & 2.113 & & 1.113 \\
2 & Comprador ocasional & 567 & 39 & 1.000 & 1.113 & \\
3 & Comprador esporádico & 446 & 31 & & 2.113 & 1.000 \\
\hline
\end{tabular}

Fuente: elaboración propia.

Los resultados del análisis clúster sugieren tres clasificaciones de compradores electrónicos en función de su frecuencia de consumo. El primero de ellos (grupo 1), aunque se sitúa como el grupo con menos individuos (441), es el que muestra mayor frecuencia de consumo electrónico (alrededor de una vez al mes). El segundo grupo (grupo 2), con una frecuencia de consumo electrónico de al menos dos veces por año, destaca como el 
grupo que integra más personas (567). Finalmente, el grupo 3 es el segundo más numeroso (446 personas) que, de acuerdo con la variable de identificación, es el que manifiesta menor frecuencia de consumo (alrededor de una vez al año).

Una vez identificados, se definieron estos tres grupos de compradores electrónicos con base en las características sociodemográficas y conductuales de sus miembros. A continuación se presenta el perfil de cada uno de ellos.

\section{1. Comprador frecuente (grupo 1)}

Supone el grupo más pequeño, representa 30\% de los compradores electrónicos y se caracteriza por concentrar a quienes compran por internet con mayor frecuencia (al menos una vez al mes). Está conformado principalmente por hombres (60\%) de 33 años en promedio, con estudios de licenciatura y posgrado (64\%).

El comprador frecuente usa diariamente la computadora (84\%). En promedio, pasa frente a la computadora 5.7 horas al día. El comprador frecuente emplea la computadora por motivos profesionales $(74 \%)$ y como medio para capacitarse (48\%). Sin embargo, el principal uso que le dan es de ocio y entretenimiento (83\%).

Este tipo de comprador registra acceso diario a internet y en promedio asigna 6.6 horas diarias a navegar (una hora más que el consumidor esporádico). El teléfono inteligente es el dispositivo a través del cual navega casi la totalidad de los compradores frecuentes (95\%).

Los compradores frecuentes usan internet para proveerse de información en general (96\%), enviar y recibir mensajes instantáneos (Whatsapp, Messenger, Skype) (96\%), enviar y recibir correos (93\%), acceder a redes sociales (93\%), observar a contenidos audiovisuales gratuitos (YouTube, Vimeo, etcétera) (90\%), descargar juegos y música (88\%), leer periódicos, libro o revistas (78\%), informarse sobre salud (78\%), descargar software (59\%), entablar conversaciones telefónicas (67\%) y escuchar contenidos de audio [Spotify] (65\%). No obstante, este segmento se caracteriza por incorporar a sus hábitos de internet la realización de operaciones bancarias (51\%) y mirar a contenidos audiovisuales de pago (Netflix, Clarovideo, iTunes) (53\%).

En cuanto a los hábitos de consumo electrónico, el comprador frecuente se sitúa entre los que más riesgo monetario asumen. En promedio, la compra más alta que han hecho a través de medios electrónicos es de $\$ 5000$ pesos (36\% superior a la compra media de los compradores esporádicos). Sus compras las realizan principalmente en páginas web nacionales (42\%). Los compradores frecuentes adquieren bienes y servicios personales $(60 \%)$, reservaciones y adquisición de boletos (52\%), aparatos electrónicos (39\%) y programas y aplicaciones (35\%).

\section{2. Comprador ocasional (grupo 2)}

Supone el grupo más numeroso, representa 39\% de los compradores electrónicos. Sus miembros manifiestan una frecuencia de compra electrónica de al menos dos veces al año. Se compone principalmente de hombres (57\%) de 33 años en promedio, con estudios de licenciatura y posgrado (63\%).

Estos compradores utilizan diariamente la computadora (80\%) con un promedio de hasta 5.3 horas al día. Como ocurre en el grupo anterior, al margen del uso para el trabajo (75\% utiliza la computadora para cuestiones laborales), los compradores ocasionales usan la computadora principalmente para ocio y entretenimiento (83\%).

Los compradores ocasionales registran acceso diario a internet. En promedio asignan 6 horas diarias a navegar por internet; el teléfono inteligente es el dispositivo que más utilizan para navegar (93\%). Respecto a sus dinámicas en internet, estos compradores lo emplean sobre todo para informarse y comunicarse: obtener información en general (97\%), enviar y recibir mensajes instantáneos (Whatsapp, Messenger, Skype) (97\%), enviar y recibir correos (95\%), leer periódicos, libros o revistas (78\%), informarse sobre salud (76\%), entablar conversaciones telefónicas (67\%) y crear o visitar blogs (51\%), con fines de ocio y entretenimiento (acceder a 
redes sociales) (93\%), acceder a contenidos audiovisuales gratuitos (YouTube, Vimeo, etcétera) (90\%), descargar juegos y música (87\%) y escuchar contenidos de audio (Spotify) (63\%). Si algo caracteriza a este grupo, es el uso que le dan a internet como medio de comunicación con el Gobierno. Así pues, 38\% emplea el internet para interactuar con el Gobierno, 52\% descarga formatos y $45 \%$ llena y envía formatos oficiales.

Respecto a los hábitos de consumo electrónico, el comprador ocasional asume el mismo riesgo monetario que los compradores frecuentes: en promedio, la compra más alta se sitúo en torno a los $\$ 5000$ pesos. Sus compras las realizan en páginas web nacionales (57\%), adquieren bienes y servicios personales (52\%) y reservaciones y adquisición de boletos (44\%).

\section{3. Comprador esporádico (grupo 3)}

Este grupo aglutina $31 \%$ de los compradores electrónicos y se caracteriza por concentrar a aquellas personas con menor frecuencia de consumo (alrededor de una vez al año). Se compone de hombres y mujeres ( $54 \%$ y $46 \%$ respectivamente) de 32 años en promedio, con estudios de licenciatura y posgrado (50\%).

El comprador esporádico hace uso diario de la computadora (71\%) en promedio 5 horas al día. Aunque también emplea la computadora para trabajar (63\%) y para capacitarse (41\%), el principal uso que le da es como medio de entretenimiento (71\%).

Estos compradores usan a diario el internet (90\%) con una media de 5.6 horas. La mayoría de ellos navega en teléfonos inteligentes (91\%) y lo hacen principalmente para proveerse de información en general (95\%), enviar y recibir correos (92\%), enviar y recibir mensajes instantáneos (Whatsapp, Messenger, Skype) (92\%), revisar redes sociales (91\%), acceder a contenidos audiovisuales gratuitos (YouTube, Vimeo, etcétera) (88\%), descargar juegos y música (85\%), leer periódicos, libros, o revistas (74\%), informarse sobre salud (73\%), descargar software (59\%), acceder a contenidos de audio (Spotify) (59\%) y entablar conversaciones telefónicas (58\%).

En lo relativo a sus dinámicas de consumo electrónico, el comprador esporádico es el grupo que menor riesgo monetario asume. En este aspecto, en promedio, la compra más alta alcanzó los \$3200 pesos. La mayor parte de sus adquisiciones las hace en páginas web nacionales (66\%). Entre sus consumos destaca la adquisición de bienes y servicios personales (46\%) y la reservación y adquisición de boletos (29\%).

Una vez identificados los compradores electrónicos con base en la frecuencia de consumo, el interés del trabajo se centró en analizar si las diferencias en el consumo se pueden atribuir a las experiencias negativas experimentadas por los consumidores (problemas en la entrega, la seguridad del pago, la seguridad de los datos u otros). Para esto, se llevó a cabo un análisis de tablas de contingencia que permitió identificar la dependencia entre las variables. Si bien las tablas analizan la dependencia o no entre variables categóricas, éstas no indican la relación que existe entre las categorías de las variables. Adicionalmente, se realizó un análisis de correspondencias con el propósito de identificar la relación entre categorías.

Los resultados de las tablas de contingencia muestran que existe una relación entre los diferentes segmentos de mercado y las experiencias negativas experimentadas (cuadro 3 y cuadro 4). Sin embargo, esta relación sólo es patente cuanto se trata de experiencias negativas relacionadas con la entrega del bien adquirido. Así pues, durante el proceso de compra electrónica, el hecho de que el comprador haya experimentado problemas con la entrega del producto guarda relación con los segmentos identificados, que a su vez representan diferentes frecuencias de consumo $(P h i=0.319$, Sig. $=0.007$; V de Cramer $=0.319$, Sig. $=0.007$, Coeficiente de contingencia $=0.304$, Sig. $=0.007)$. En el cuadro 4 se muestran los resultados del chi cuadrado y la razón de verosimilitud que confirman la relación genuina entre estas variables.

Sin embargo, por medio de las tablas de contingencia no es posible identificar con qué segmento(s) en concreto se relacionan los problemas de entrega. Por esta razón, se llevó a cabo un análisis de correspondencia con la finalidad de identificar la relación entre los segmentos (que representan frecuencia de compra) y los problemas con la entrega del producto adquirido. 


\section{CUADRO 3}

Tabla de contingencia (estadísticos)

\begin{tabular}{|lcccccc}
\hline \multicolumn{7}{c}{ Estadísticos } \\
\hline Tipo de problema & Lambda & $\begin{array}{c}\text { Tau de Goodmand y } \\
\text { Kruskal }\end{array}$ & $\begin{array}{l}\text { Coeficiente de } \\
\text { incertidumbre }\end{array}$ & Phi & V de Cramer $\begin{array}{c}\text { Coeficiente de } \\
\text { Contingencia }\end{array}$ \\
\hline Entrega & 0.217 & $0.102^{* *}$ & $0.076^{* *}$ & $0.319^{*}$ & $0.319^{* *}$ & $0.304^{* *}$ \\
\hline $\begin{array}{l}\text { Seguridad del pago } \\
\text { Seguridad de los } \\
\text { datos personales }\end{array}$ & 0.000 & 0.012 & 0.011 & 0.108 & 0.108 & 0.107 \\
$\begin{array}{l}\text { Otro tipo de } \\
\text { problemas }\end{array}$ & 0.000 & 0.001 & 0.002 & 0.033 & 0.033 & 0.033 \\
\hline
\end{tabular}

Fuente: elaboración propia.

Nota: ${ }^{* *}$ significativo a $95 \%$.

\section{CUADRO 4}

Tabla de contingencia (significancia)

\begin{tabular}{|llccc}
\hline Tipo de problema & & Valor & $g l$ & Sig. \\
\hline Relacionado con la entrega & chi cuadrado de Pearson & 9.872 & 2 & 0.007 \\
& Razón de verosimilitud & 10.261 & 2 & 0.006 \\
Relacionado con la seguridad & chi cuadrado de Pearson & 1.133 & 2 & 0.567 \\
del pago & Razón de verosimilitud & 1.164 & 2 & 0.559 \\
Relacionado con la seguridad & chi cuadrado de Pearson & 0.106 & 2 & 0.984 \\
de los datos personales & Razón de verosimilitud & 0.109 & 2 & 0.947 \\
Otro tipo de problemas & chi cuadrado de Pearson & 2.076 & 2 & 0.354 \\
& Razón de verosimilitud & 2.031 & 2 & 0.362 \\
\hline
\end{tabular}

Fuente: elaboración propia.

El cuadro 5 muestra los resultados del análisis de correspondencia, los cuales reflejan una dimensión que explica $100 \%$ de la varianza/inercia (chi cuadrado $=9.872$, Sig. $=0.007$ ). De este modo, se asume que las frecuencias de consumo electrónico representadas por los clúster son distintas respecto a si tuvieron problemas relacionados con la entrega del producto.

\section{CUADRO 5}

Análisis de correspondencias

\begin{tabular}{|ccccccc}
\hline \multirow{2}{*}{ Dimensión } & & & & & \multicolumn{2}{c}{ Proporción de inercia } \\
\cline { 6 - 7 } & Valor propio & Inercia & chi cuadrado & Sig. & Explicada & Acumulada \\
\hline 1 & 0.319 & 0.102 & 9.872 & 0.007 & 1.000 & 1.000 \\
\hline
\end{tabular}

Fuente: elaboración propia. 
Concretamente, los resultados del análisis de correspondencias muestran que los compradores ocasionales, aquellos que hacen compras de al menos dos veces al año, están relacionados con problemas en la entrega de los productos adquiridos a través de internet $(-0.826$ y -0.536 respectivamente), mientras que los compradores frecuentes, quienes presentan la mayor frecuencia de consumo (al menos una vez al mes), lo están con la categoría de no haber presentado problemas durante el proceso de compra electrónica ( 0.547 y 0.595 respectivamente). En resumen, de acuerdo con los resultados, la frecuencia de consumo electrónico (representada por las tipologías de comprador descritas), se relaciona con las experiencias previas. De manera específica, los eventos negativos como experimentar problemas relacionados con la entrega del producto disminuye la frecuencia de compra: pasa de al menos una vez al mes (compradores frecuentes) a dos veces al año (compradores ocasionales).

\section{Conclusiones y Prospectiva}

Este artículo contribuye especialmente al conocimiento sobre la actividad de búsqueda como parte del proceso de compra online y el efecto de la experiencia previa en la frecuencia de compra en consumo de aparatos electrónicos. Los datos obtenidos de una muestra de 1495 consumidores electrónicos mexicanos (INEGI, 2015), además de la tipología, encuentra que el consumo describe individuos, primordialmente hombres, con una media de 32 años de edad, con estudios de licenciatura y posgrado, cuyas compras se inclinan a bienes y servicios personales y reservaciones y boletos. Estos individuos se han clasificado de acuerdo con la frecuencia de compra en $a$ ) frecuentes, $b$ ) ocasionales y $c$ ) esporádicos, los cuales parecen seguir comportamiento de $a$ ) profesionales, $b$ ) laborales y $c$ ) sociales respectivamente. Los compradores frecuentes y ocasionales realizan compras por importes alrededor de los 250 dólares americanos, en cuyo proceso buscan información en internet durante el tiempo laboral, lo complementan con el tiempo personal y finalizan la compraventa mediante un teléfono móvil en su mayoría.

Es evidente cómo influye la experiencia previa negativa - relacionada con la entrega del bien adquirido-en la frecuencia de compra online, puesto que implica un riesgo percibido para los compradores ocasionales. Los compradores frecuentes realizan usualmente pagos desde sus teléfonos móviles y los ocasionales acostumbran comunicarse e interactuar con el Gobierno, pero los hábitos de compra se asemejan a los compradores frecuentes; con esto se esperaría que la experiencia previa positiva aumenta la frecuencia de compra de los tres tipos de compradores.

La contribución principal de este artículo es la identificación de tres grupos de consumidores electrónicos (habituales, ocasionales y esporádicos), lo cual prueba que no hay homogeneidad para este tipo de usuarios. Incluso, podría decirse que los compradores frecuentes que tuviesen experiencia previa positiva tendrían compras más habituales y ampliarían la proporción de clientes frecuentes. En cuanto al hallazgo del uso de teléfono móvil para efectuar la compraventa B2C, pese a que el estudio aborda la compra por internet realizada para el negocio, el porcentaje de internautas que compran es superior al reportado para España en el estudio de San Martín y López (2010).

Como implicaciones gerenciales, destaca la necesidad de mejorar el servicio offline para mejorar la percepción, confianza y frecuencia de compras en internet. Un reto particular es la incorporación de mejoras tecnológicas en las pantallas de los instrumentos B2C, el diseño de las páginas web, las políticas de seguridad y privacidad y, por otro lado, las características de los servicios ofrecidos, el método de pago, los límites de compra, la presentación de los productos, la información proporcionada tal como aseguran Vrechopoulos et al. (2001).

En cuanto a las limitaciones, se tiene en primer momento la información sólo en México, por lo que no se pueden generalizar los resultados. Adicionalmente, otra limitación es el enfoque cualitativo de variables de percepción y la omisión de variables como confianza, riesgo percibido o experiencia interactiva que influyen de manera importante en la compra electrónica. 


\section{REFERENCIAS}

Aljukhadar, M., \& Senecal, S. (2011). Segmenting the online consumer market. Marketing Intelligence \& Planning, 29(4), 421-435. https://doi.org/10.1108/02634501111138572.

Allred, C., Smith, S., \& Swinyard, W. (2006). E-shopping lovers and fearful conservatives: A market segmentation analysis. International Journal of Retail \& Distribution Management, 34(4/5), 308-333. https:// doi.org/10.1108/09590550610660251.

AMIPCI (Asociación Mexicana de Internet). (2017). Estudio de comercio electrónico en México 2016. Disponible en https://www.asociaciondeinternet.mx/es/.

Anderson, R. E., \& Srinivansan, S. S. (2003). E-satisfaction and e-loyalty: A contingency framework. Psychology and Marketing, 20(2), 123-138.

Barnes, S., Bauer, H., Neumann, M. \& Huber, F. (2007). Segmenting cyberspace: A customer typology for the internet. European Journal of Marketing, 41(1/2), 71-93. http://dx.doi.org/10.1108/03090560710718120

Becerra, E. P., \& Korgaonkar, P. K. (2011). Effects of trust beliefs on consumers' online intentions. European Journal of Marketing, 45(6), 936-962.

Bhatnagar, A. \& Ghose, S. (2004). Segmenting consumers base don the benefits and risks of Internet shopping. Journal of Business Research, 57(12), 1352-1360. https://doi.org/10.1016/S01482963(03)00067-5.

Bigné, E., Ruiz, C., \& Sanz, S. (2005). The impact of internet user shopping patterns and demographics on consumer mobile buying behavior. Journal of Electronic Commerce Research, 6(3), 193-209.

Brengman, M., Geuens, M., Weijters, B., Smith, S., \& Swinyard, S. (2005). Segmenting internet shoppers based on their web-usage-related lifestyle: A cross-cultural validation. Journal of Business Research, 58, 79-88. https://doi.org/10.1016/S0148-2963(02)00476-9.

Chen, Y., Kuo, M., Wu, S., \& Tang, K. (2009). Discovering recency, frequency, and monetary (RFM) sequential patterns from customers' purchasing data. Electronic Commerce Research and Applications, 8, 241-251. https://doi.org/10.1016/j.elerap.2009.03.002.

Chiou, J., \& Pan, L. (2009). Antecedents of internet retailing loyalty: Differences between heavy versus light shoppers. Journal of Business and Psychology, 24(3), 327-339.

Chong, A. Y. (2013). Mobile commerce usage activities: The roles of demographic and motivation variables. Technological Forecasting and Social Change, 80(7), 1350-1359. https://doi.org/10.1016/j.techfore.2012.12.011.

Christodoulides, G., Michaelidou, N., \& Siamagka, N. (2013). A typology of internet users based on comparative affective states: Evidence from eight countries. European Journal of Marketing, 47(1/2), 153-173. https://doi.org/10.1108/03090561311285493.

Cristóbal Fransi, E., Daries Ramon, N., \& Baldomar, J. (2014). Segmentación de los e-consumidores: un estudio aplicado a partir de las perspectivas de uso de Internet. Cuadernos de Gestión, 14(1), 33-55.

Donthu, N., \& Garcia, A. (1999). The Internet Shopper. Journal of Advertising Research, 39, 52-58.

Ganesh, J., Reynolds, K., Luckett, M., \& Pomirleanu, N. (2010). Online shopper motivations, and e-store attributes: An examination of online patronage behavior and shopper typologies. Journal of Retailing, 86(1), https://doi.org/10.1016/j.jretai.2010.01.003.

Hernández, B., Jiménez, J., \& Martín, M. J. (2011). Age, gender and income: Do they really moderate online shopping behaviour? Online Information Review, 35(1), 113-133. 
Hill, W., Beatty, S., \& Walsh, G. (2013). A segmentation of adolescent online users and shoppers. Journal of Services Marketing, 24(5), 347-360. https://doi.org/10.1108/JSM-10-2011-0157.

Iacobucci, D., \& Churchill, G. (2015). Marketing research: Methodological foundations. Nashville: Earlie LiteBooks.

INEGI (Instituto Nacional de Estadística y Geografía). Encuesta Nacional sobre Disponibilidad y uso de TIC en Hogares ENDUTIH (2015). Disponible en http://www.beta.inegi.org.mx/proyectos/enchogares/ regulares/dutih/2015/default.html.

Joia, L. A., \& Sanz, P. S. (2006). The financial potential of sporadic customers in E-Retailing: Evidence from the brazilian home appliance sector. Journal of Electronic Commerce in Organizations, 4(1), 18-32.

Kau, A., Tang, Y., \& Ghose, S. (2003). Typology of online shoppers. Journal of Consumer Marketing, 20(2), 139-156.

Klaus, P. (2013). The case of amazon.com: Towards a conceptual framework of online shoppers service experience (OCSE) using the emerging consensus technique (ECT). Journal of Service Marketing, 27(6), 443-457.

Liu, Y., Li, H., Peng, G., Lv, B., \& Zhang, C. (2015). Online purchaser segmentation and promotion strategy selection: evidence from Chinese e-commerce market. Ann Oper Res, 233, 263-279. https://doi. org/10.1007/s10479-013-1443-z.

Martin, J., Mortimer, G., \& Andrews, L. (2015). Re-examining online customer experience to include purchase frequency and perceived risk. Journal of Retailing and Consumer Services, 25, 81-95.

Min, S., Overby, J. W., \& Im, K. S. (2012). Relationships between desired attributes, consequences and purchase frequency.Journal of Consumer Marketing, 29(6), 423-435. https://doi.org/10.1108/07363761211259232.

Pappas, I., Pateli, A., Giannakos, M., \& Chrissikopoulos, V. (2014). Moderating effects of online shopping experience on customer satisfaction and repurchase intentions, International Journal of Retail \& Distribution Management, 42(3), 187-204. https://doi.org/10.1108/IJRDM-03-2012-0034.

Prodanova, J., y San Martín Gutiérrez, S. (2013). Estudio sobre el impacto del género y las emociones en el comportamiento de compra online de viajes. Revista de análisis turístico, 2.

Punj, G., \& Stewart, D. (1983). Cluster analysis in marketing research: Review and suggestions for application. Journal of Marketing Research, 20, 134-48.

Raju, C. V. L., Narahari, Y., \& Ravikumar, K. (2006). Learning dynamic prices in electronic retail markets with customer segmentation. Annals of Operations Research, 143(1,), 59-75. https://doi.org/10.1007/ s10479-006-7372-3.

Rodríguez-Torrico, P., San Martín, S. y San José, R. (2012). Múltiples formas para segmentar el mercado de compradores online y móvil. Cuadernos de Estudios Empresariales, 22, 99-128. http://dx.doi.org/10.5209/ rev_CESE.2012.v22.44647.

Rohm, A., \& Swaminathan, V. (2004). A typology of online shoppers base on shopping motivations. Journal of Business Research, 57, 748-757. https://doi.org/10.1016/S0148-2963(02)00351-X.

Rose, S., Clark, M., Samouel, P., \& Hair, N. (2012). Online customer experience in e-retailing: An empirical model of antecedents and outcomes. Journal of Retail, 88(2), 308-322. https://doi.org/10.1016/j. jretai.2012.03.001.

Ruiz Mafé, C. y Sanz Blas, S. (2005). Segmentación del comprador a distancia: un análisis de los medios internet y televisión. Investigaciones Europeas de Dirección y Economía de la Empresa, 11(2), 163-182.

San Martín, S. y López, B. (2010). Posibilidades de la compraventa B2C por teléfono móvil en comparación con Internet. Cuadernos de Gestión, 10(1), 17-34. 
Sheehan, K. (2002). Toward a typology of internet users and online privacy concerns. The information Society, 18, 21-32.

Statista. (2017). Retail e-commerce sales worldwide from 2014 to 2021 (in billion U.S. dollars). Consultado el 16 de octubre de 2017. Disponible en https://www.statista.com/statistics/379046/worldwide-retail-e-commerce-sales/.

Swinyard, W., \& Smith, S. (2003). Why people don't shop online: A lifestyle study of the internet consumer. Psychology \& Marketing, 20(7), 567-597. https://doi.org/10.1002/mar.10087

Vrechopoulos, A., Siomkos, G., \& Doukidis, G. (2001). Internet shopping adoption by Greek consumers. European Journal of Innovation Management, 4(3), 142-153. http://dx.doi.org/10.1108/14601060110399306.

Wu, R., \& Chou, P. (2011). Customer segmentation of multiple category data in e-commerce using a soft-clustering approach. Electronic Commerce Research and Applications, 10(3), 331-341. https://doi. org/10.1016/j.elerap.2010.11.002.

Yang, K., \& Hye-Young, K. (2012) Mobile shopping motivation: An application of multiple discriminant analysis. International Journal of Retail \& Distribution Management, 40(10), 778-789. https://doi. org/10.1108/09590551211263182.

Zhou, L., Dai, L., \& Zhang, D. (2007). Online shopping acceptance model-a critical survey of consumer factors in online shopping. Journal of Electronic Commerce Research, 8(1), 41-62.

\section{Notas}

[1] En los últimos doce meses, ¿ha realizado compras por internet? Excluya compras por motivos de trabajo e incluya las realizadas para su negocio.

[2] Previo al análisis clúster no jerárquico, se realizó un análisis clúster jerárquico con la finalidad de conocer el número óptimo de conglomerados existente en los datos empleados. Los resultados muestran que el número adecuado de conglomerados dentro de los datos se sitúa en torno a dos y tres grupos.

\section{BY-NC-ND}

